\title{
Effectiveness of a nurse facilitated cognitive group intervention among mild to moderately-depressed-women in KwaZulu-Natal, South Africa
}

\section{Chetty', ME Hoque ${ }^{2}$}

1Department of Nursing Science, School of Health Care Sciences, University of Limpopo (Medunsa Campus), South Africa 2Department of Public Health, School of Health Care Sciences, University of Limpopo (Medunsa Campus), South Africa

\begin{abstract}
Objective: The purpose of the study was to determine the effectiveness of a nurse-facilitated-cognitive-group (NFCG) intervention as an adjunct to antidepressant medication, in mild to moderately, depressed women. Method: This was a quasi-experimental, nonequivalent, control group design study. A sample of 30 consenting participants was selected from an urban, community psychiatric clinic, and the participants were randomly allocated to the control and the intervention groups. The effectiveness of the intervention was measured using the Beck Depression Inventory (BDI). Results: After six weeks of implementation of the NFCG intervention, there was a decrease in the BDI scores of the intervention group, and an increase in the BDI scores in the control group (CG) - but the difference in scores was not significant (Student's t-test=1.076, p=0.291). After 12 weeks of the group intervention, the BDI scores for the intervention group, showed a considerable reduction in their levels of depression, whilst the participants of the control group had a further increase in their scores. There was a statistically significant difference between the groups, with respect to the BDI scores $(p<0.001)$. The Friedman test indicated that the mean BDI scores, were statistically significant $(p<0.001)$ within the intervention group, meaning that the BDI scores improved, at the end of the intervention for all the participants. Analysis of the BDI scores, using the Friedman test, showed that there was no improvement in the control group ( $p=0.597)$. Conclusion: The NFCG intervention, as an adjunct to antidepressant medication, contributed to a reduction in depressive symptoms.
\end{abstract}

Keywords: Psychiatric nursing; Psychotherapy, Group; Cognitive Behavior Therapy; South Africa

Received: 10-05-2011

Accepted: $04-11-2011$

doi: http://dx.doi.org/10.4314/ajpsy.v16il.5

\section{Introduction}

Depression is a mood disorder, commonly presenting symptomatically as a feeling of sadness, loneliness, inadequacy, helplessness, poor-morale and low self-esteem. ${ }^{1}$ Depressed patients experience both emotional and psychological problems, are extremely vulnerable, and endure difficulties due to the disease process. A variety of perceived, beneficial, "alternative" interventions, external to the "mainstream, health-care system" are sometimes explored by these depressed patients, as health-seekingmeasures. ${ }^{2}$ Approximately 15-20\% of depressed patients commit suicide. ${ }^{3}$ Depression has been noted to induce suicidal ideation whilst a history of previous suicidal attempts increases the risk for suicide. ${ }^{4}$ Depression may occur in persons of any age, gender or background within a person's lifetime. In the year 2000, depression was the leading cause of disability, as measured by 'years of life lived with disability', and the 4th leading contributor to the global burden of disease, 'disability adjusted life years lost' (DALY's). ${ }^{3}$ By the year 2020, depression is projected to reach the 2 nd ranking of DALY's, calculated for all ages and both genders. ${ }^{3}$

Empirical support for Cognitive Behaviour Therapy (CBT) as an intervention is growing. ${ }^{5}$ CBT versus pharmacotherapy has many advantages, including reduced patient dropouts, reduced relapses, reduced side effects, and an improvement in patient satisfaction. Treatment with psychiatric medication as compared to CBT requires 
continuous administration and compliance to be effective. ${ }^{5}$ CBT provides a "time-limited", "empirically-grounded", "evidenced based treatment-intervention" for use by depressed patients. ${ }^{6}$ It is effective in treating acute depression, has prophylactic effects and is accessible and adaptable to different population groups and settings. CBT is known as the "mainstay approach to depression". ${ }^{6}$ Various studies have also noted the cost effectiveness of CBT compared to other interventions for the treatment of major depression. , $^{1}$

The benefit of group involvement is well-understood by psychiatric nurses. Efficiency, cost-effectiveness and efficacy are some known benefits of group activities. Group environments facilitate trust and safety in enhancing learning and practising of new behaviours. ${ }^{8}$

The training of psychiatric nurses in CBT has been found to be cost-effective. Teaching the requisite competencies and the essentials of psychotherapy, whilst maintaining the total credit-load of the existing nursing curricula is a challenge for nursing education. Psychiatric nurse specialists have been practicing psychotherapy since $1954 .{ }^{9}$ Psychotherapy, of which CBT is an example, has been identified as a necessary competency and skill required by all psychiatric, mental, health-nurse practitioners. ${ }^{9}$ However, the position of the advanced practice psychiatric nurse in South Africa, needs to be clearly defined, accepted and recognized by the regulatory bodies and mental health practitioners. Specialists such as psychiatrists and psychologists are not always available at many public health clinics, and often psychiatric nurses are expected to perform some tasks, that are normally performed by these specialists. ${ }^{10,11}$

The current status of mental health is low within the public health-system in South Africa due to the concomitant lowstaffing of mental health practitioners and high patient-ratios both in the urban and rural psychiatric institutions. ${ }^{12}$ Increased workloads and an increased number of patients in the public sector, has caused over-crowding, contributing to the low-morale and the negative image of South African psychiatric nurses. ${ }^{12}$ High patient numbers has increased the workload and has impacted negatively on patient care and nurses' job-satisfaction. $1^{2}$ There is an increasing interest in the role of CBT in mental health. ${ }^{5}$ The current underuse of CBT remains a concern within the mental health service. CBT has both short and long-term advantages. These include lack of side effects and the prevention of relapses due to recurrent depression. 5,6

Little research has been conducted in South Africa on the effectiveness of NFCG intervention. A study has concluded that the Beck Depression Inventory (BDI) scale, is feasible as a valid alternative to the interview, and especially useful as a diagnostic tool in mental, health-services, with limitedstaffing and time restraints. ${ }^{13}$ Therefore, the objective of this study is to determine the effectiveness of a NFCG intervention as an adjunct to antidepressant medication on mild to moderately depressed Indian women, attending an urban-community-psychiatric-clinic in southern KwaZuluNatal, over a fixed period of time. The BDI score is used for inclusion of the sample as well as to measure the effectiveness of the CBT. The study describes the comparison of the BDI scores at pre-test, post-testl and posttest2.

\section{Methods}

\section{Study design and setting}

This was a quasi-experimental, non-equivalent, controlgroup design study. This study used quantitative methods in the research design, represented though the evaluation of the numeric data in terms of the level of depression. A Pretest and two sequential Post-tests were undertaken at 6 weeks and 12 weeks respectively. Comparisons of the BDI scores were used to compare outcomes between the specific group intervention (independent variable) and the control group, who were treated conventionally, that is, no psychotherapeutic intervention, only standard treatment (the "usual treatment"), incorporating the use of antidepressant medication (dependent variable)

The study setting was a public, community, psychiatric clinic, serviced by the KwaZulu-Natal Provincial Health Services, for clients residing in specific areas of the Durban South Metropolitan Region. This clinic studied was one of the busiest, urban community, psychiatric clinics in southern, KwaZulu-Natal. The clinic attendance ranges from 100 to 150 patients daily. A monthly, maximum number of 3000 patients were seen at this public, psychiatric-clinic, compared with a monthly average of 70 to 1,800 patient attendances at other clinics in this region. ${ }^{14}$ This clinic was extremely busy and operated from Mondays to Fridays (5days/week), from $07 h 30$ to 16h00. The mornings were very busy, as the medical and pharmacy staffs were only available from about 08 h00 until midday daily (12h00). In the absence of the medical and pharmacy staff, the psychiatric nurses continued rendering follow-up-treatment and attended to emergency patients. The clinic was relatively well-staffed with multi-disciplinary team (MDT) members; one clinical psychologist, three psychiatrists, who worked on three different morning sessions and three psychiatric medical officers, who worked in the mornings only, and were also allocated on a sessional, rotational basis to other satellite psychiatric clinics in this region. The nursing personnel were eight (8) full-time nursing staff, comprising of one (1) auxiliary nurse, two (2) enrolled nurses, trained in practical general nursing only, and five (5) professional, registered psychiatric nurses. No social workers were available at the clinic. Referrals were made to the specific social worker, who consulted from a nearby office away from the psychiatric clinic. Appointments were made telephonically or by referral letter with the Social Worker's office. Exceptions were made in obtaining urgent appointments in crisis situations. There was also an occupational therapy department adjoining the clinic, with a staff complement of three (3) registered occupational therapists and one (1) occupational therapy assistant.

The clinic services were rendered mainly by psychiatric nurses. The role and functions of all the nurses were according to clinic procedures, the Department of Health (KZN) duty sheet (Z249) and the South African Nursing Council's Regulation, Scope of Practice R2598, and the Nursing Act 33 of 2005. The roles of the nursing staff included: screening of new patients, reviewing of follow-uppatients attending to crises and referrals for in-patient admission. The follow-up or "repeat", "usual or standard treatment" patients had a monthly appointments to collect their monthly medication, were interviewed by registered 
nurses, were given a follow-up date for the next appointment, and referred to the multidisciplinary members if problems such as relapse, admission, suicide or major side effects were identified during the interview. The registered psychiatric nurses attended to patients on their specified, monthly, clinic appointments, acted as consultants and resource persons for the community, general hospital and primary health clinics, provided advice and assistance, referred patients to other agencies and community resources, undertook individual and family counselling, collected all blood samples from patients as prescribed, delivered blood samples to the laboratories, collected blood results, conducted home-visits for bed-bound or disabled patients and attended to all patient emergencies in the absence of medical staff and transported patients to hospitals.

\section{Sample}

The study sample comprised of depressed, Indian South African-women. The accessible population consisted of Indian women who were already diagnosed with depression, were currently on antidepressant medication for a continuous period of three months or more, which was received from the community psychiatric clinic in this study. The researcher was able to ascertain from prospective clinics records, that 117 women were receiving antidepressant medication, over a continuous period of more than three (3) month.s.

\section{Sampling and Sample size}

Convenience sampling was used. Thirty (30) consenting participants were selected and randomly allocated to the two groups. Group one was designated as the intervention group and group two as the control group. Each group had 15 participants respectively. The number of participants was determined on the basis of the optimal size in a group, ranging from a minimum of five (5) to a maximum of 15 members. ${ }^{15,16}$ The total sample size of 30 was large enough, to allow for limited attrition, voluntary withdrawal, persistent non-attendance, illness and crises. In fact, the sample of 30 participants was retained throughout the study.

\section{Inclusion criteria}

In order to be included in this study, the women had to:

- speak and understand English,

- be Indian women,

- reside in the specified geographical areas in the south of Durban as prescribed by the study,

- be between the ages of 25 to 65 years,

- be diagnosed as depressed, either by clinical features or by DSM IV diagnosis, by a Medical Officer or a Psychiatrist employed at the clinic,

- have a Beck Depression Inventory score of more than nine (9) and less than 29 (mild to moderate depression),

- have been on antidepressant medication prescribed at the clinic by the Psychiatrist or the Medical Officer for three months or more. ${ }^{17,18}$

In addition, the participants were assessed by a psychiatric nurse on a monthly basis or by a psychiatric clinic doctor if a script needed to be reviewed or if the patient had problems requiring the doctor's attention and had to collect the antidepressant medication from the clinic pharmacy. They also had to be on the clinic register records to confirm their continuous attendance and had to agree to attend all the group sessions during the study.

\section{Recruitment/selection}

A poster - displayed six (6) weeks prior to the study commencement - informed female patients with a diagnosis of depression attending the clinic, about the proposed study and its purpose. The poster was placed strategically on the notice board in the clinic's waiting area. Verbal explanations and information were given to patients who expressed interested in this study. The multidisciplinary team, consisting of nursing staff, social workers, occupational therapists, clinical psychologists, psychiatrists, medical officers and student nurses assisted in recruiting participants for this study.

To assign participants in different groups, 30 cards which were kept in a hat with coded alphabets $(\mathrm{C} / \mathrm{G}$, N/FC/G), were folded, and were picked up by each individual who satisfied the inclusion criteria. Cards were made using capital letter ' $\mathrm{C} / \mathrm{G}$ ' representing the Control group, and capital letters 'N/F/C/G' the nurse-facilitatedcognitive group. Once the participants were assigned, they were told to attend the three scoring sessions, which were held six (6) and twelve (12) weekly respectively.

\section{Instruments}

The researcher formulated and developed the Personal Profile questionnaire (PPQ) instrument. The objective of the $\mathrm{PPQ}$ was to identify differences and similarities in the participant's individual profiles (i.e. demographic details) and to assess the inclusion criteria for selection into the study. The PPQ questionnaire comprised of the following items: Marital, Educational and Employment status, Age (in years), and current Antidepressant medication use. The severity of depressive symptoms was scored based on the BDI Scale. The BDI scale was used for inclusion as well as for effectiveness of the intervention program. The BDI scale is a validated instrument. The BDI Scale was initially designed, to measure the severity or depth of depression, in psychiatric patients after the clinical diagnosis had been made, but later was used for screening in the community and for clinical research, where it was found to have performed well. ${ }^{13,18}$ It is a recognized and commonly used scale and has been used in over 500 reported studies. ${ }^{19}$ In this study, BDI scores between 10 and 28 were considered for inclusion as mild to moderate depression.

A pilot study was undertaken on 10 other female depressed patients at a similar psychiatric clinic facility in the region, prior to the main study, for reliability, pre-testing and accuracy purposes.

\section{The group intervention}

The nurse-facilitated-cognitive-group (NFCG) intervention followed the principles of the cognitive group therapy programme, as indicated in Verona Gordon's (1988) Women's Workbook and Facilitator's Manual. It was focused on the present, problem solving and was time-limited. ${ }^{20}$ This group was facilitated by an Advanced Psychiatric nurse with 
the assistance of a qualified, registered psychiatric nurse. Group sessions were held weekly on Wednesday's in the clinic, over a one to two hours duration over 15 group sessions. The intervention was applied according to the cognitive behavioural, structured group approach based on Gordon's model, as explained in the Women's Workbook and the Facilitator's Manual. ${ }^{20}$ The facilitation included information on group dynamics and reinforcement theory. The manuals contained specific lecture content, objectives, and discussion questions for each of the 15 group sessions based on the Women's Workbook. Weekly topics and issues for discussion included goal-setting, depression, self-worth, relationships, assertiveness, conflict-management, stress, nutrition and strength building, exercises. Being aware of negative thoughts, negative behaviors, learning more effective coping skills, eliminating negative defense mechanisms and gaining insight into their own dependency and helplessness was discussed. 14,21

\section{Ethics}

Ethical permission for the study was obtained from the Research, Publication and Ethics Committee of the Durban University of Technology. Permission to conduct the study was also obtained from the Director of Psychiatric Services and the Assistant Director of community Psychiatric Services, Regional Offices, KwaZulu-Natal.

Informed consent was obtained from all the participants. Participation was voluntary and the participants were allowed to withdraw from the study without any fear of discrimination. Privacy, respect, dignity, confidentiality and anonymity and confidentiality were maintained throughout the study.

\section{Data analysis}

Data were entered into a Microsoft Excel spreadsheet and exported into the SPSS version 13.0 (SPSS Inc, Chicago) for analysis. The demographic data were summarized using descriptive summary measures, expressed as mean (standard deviation) or median (minimum-maximum), for continuous variables, and percentage for categorical variables. The Students t-test was conducted to establish the relatedness or independence between the two groups, as measured at each scoring session. The Friedman test was conducted to establish if there were any significant differences in the BDI scores within each group. P-values less than 0.05 were considered statistically significant.

\section{Results}

Table I summarizes the personal profiles of the participants The mean ages of the CG control group and NFCG Intervention group were similar (45.7 years and 44.7 years respectively). The range for age was from 31 to 60 years. Regarding marital status of the women, less than half $(43.33 \%, n=13)$ were married, followed by widowed $(20.00 \%, n=6)$ and separated $(20.00 \%, n=6)$ respectively. Less than half of the participants had attended 'secondary school', (40.00\%, n=12) and 'primary school' (43.33\%, $\mathrm{n}=13$ ), whilst $10.00 \%(\mathrm{n}=3)$ had 'no formal education', and $6.67 \%(n=2)$ had completed secondary school, secretarial and pre-school-teacher-training. The majority of the participants were 'housewives' (95.55\%, n=28), whilst the

\begin{tabular}{|c|c|c|}
\hline \multicolumn{3}{|c|}{$\begin{array}{l}\text { Table l: Socio-demographic information and antidepressants } \\
\text { use by the depressed women }\end{array}$} \\
\hline Variables & $\begin{array}{l}\text { Intervention } \\
\text { group (NFCG) }\end{array}$ & $\begin{array}{l}\text { Control Group } \\
\text { (CG) }\end{array}$ \\
\hline & $n(\%)$ & $n(\%)$ \\
\hline Mean age (Range) & 44.7 yrs (31-60) & 45.7 yrs (31-58) \\
\hline $\begin{array}{l}\text { Marital status } \\
\text { Married } \\
\text { Widowed } \\
\text { Separated } \\
\text { Divorced } \\
\text { Never married }\end{array}$ & $\begin{array}{l}9(60.00) \\
1(6.66) \\
0(0) \\
4(26.66) \\
1(6.66)\end{array}$ & $\begin{array}{l}4(26.66) \\
5(33.33) \\
1(6.66) \\
2(13.33) \\
3(20.00)\end{array}$ \\
\hline $\begin{array}{l}\text { Education } \\
\text { No formal education } \\
\text { Primary school (grade 1-7) } \\
\text { Secondary school (grade 8-12) } \\
\text { Secondary school and other training }\end{array}$ & $\begin{array}{l}0(0) \\
9(60.00) \\
4(26.66) \\
2(13.33)\end{array}$ & $\begin{array}{l}3(20.00) \\
4(26.66) \\
8(53.33) \\
0(0)\end{array}$ \\
\hline $\begin{array}{l}\text { Employment status } \\
\text { Unemployed } \\
\text { Part-time formal }\end{array}$ & $\begin{array}{l}15(100) \\
0(0)\end{array}$ & $\begin{array}{l}13(86.66) \\
2(13.33)\end{array}$ \\
\hline $\begin{array}{l}\text { Antidepressants } \\
\text { Tricyclics (TCA) e.g. amitryptyline } \\
\text { Tetracyclics (TTCA) e.g. mianserin }\end{array}$ & $\begin{array}{l}10(66.76) \\
5(33.33)\end{array}$ & $\begin{array}{l}13(86.76) \\
2(13.33)\end{array}$ \\
\hline
\end{tabular}

remaining 4.45\% (n=2) had 'part-time jobs'. More than three quarters $(76.67 \%, n=23)$ of the participants were prescribed tricyclic antidepressants and the rest (23.33\%, $\mathrm{n}=7$ ) tetracyclics.

\section{Effectiveness of intervention}

An improvement in the levels of depression was denoted by a reduction in their BDI scores. The Initial scoring was at the Pre-test, the second scoring was at Post-test 1 (after six weeks), and the third scoring was at Post- test 2 (after 12 weeks).

The mean initial BDI scores of the two groups were similar. Table II shows the comparison of the mean BDI scores at the Pre-test, Post-test 1 and Post-test 2 scoring sessions between the groups. When the Initial BDI scores for the two groups were compared at Pre-test, the mean BDI scores did not significantly differ between NFGC and CG (22.4 vs 20.2, $t=1.54, p=0.135)$. This result demonstrated

Table II: The mean Beck Depression Inventory scores for the two groups at the three scoring sessions

\begin{tabular}{|l|l|l|l|}
\hline & $\begin{array}{l}\text { Intervention } \\
\text { group (NFCG) }\end{array}$ & $\begin{array}{l}\text { Control Group } \\
\text { (CG) }\end{array}$ & p value** \\
\hline Pre-test (baseline) & 22.4 & 20.2 & 0.135 \\
Post-test1 (6wk) & 17.9 & 20.7 & 0.096 \\
Post-test 2 (12 wk) & 14.6 & 21 & $<0.001$ \\
p-value* & $<0.001$ & 0.597 & \\
\hline * Friedman test, **Kruskal Wallis test & \\
\hline
\end{tabular}


that the groups were similar before the implementation of the interventions.

The mean BDI scores of the two groups after six weeks of intervention, and standard treatment, showed both a reduction and an increase in the BDI scores respectively. There was a decrease in the BDI score of the NFCG (mean $\mathrm{BDI}=17.9)$, and an increase in the BDI score in the CG (mean BDI = 20.7) (Table II). However the difference was not significant $(t=1.076, p=0.291)$.

After 12 weeks (Post-test 2) of the group interventions, the BDI scores for the participants who attended the NFCG sessions, showed a considerable reduction (mean BDI = 14.6), whilst the CG participants had an increase in their BDI scores (mean BDI = 21.0) (Table II). Using the Students t-test, there was a statistically significant difference, between the groups with respect to the mean BDI scores (14.6 vs $21.0, t=8.34, p<0.001$ ).

\section{Intra-group comparisons of BDI scores}

The initial mean BDI score was higher than the Post-test 1 and Post-test 2 score in the intervention group (22.2, 17.9, and 14.6 respectively). The Friedman test indicated that the differences in mean BDI scores were statistically significant $(p<0.001)$. However, when the mean BDI scores were analyzed using the Friedman test, there was no significant improvement in the levels of depressive symptoms in the CG (20.2, 20.7, and 21 respectively, p=0.597) (Table II).

\section{Discussion}

In this study, the effectiveness of a NFCG, as an adjunct to antidepressant medication in mild to moderately depressed Indian women, in terms of subjective findings (levels of depressive symptoms) over a fixed period of time was investigated. The NFCG demonstrated a reduction in BDI score at six weeks and with a further reduction at 12 weeks after initiating the group sessions.

There was no significant improvement regarding the levels of depressive symptoms between the groups after six weeks of the NFCG intervention. However, after 12 weeks of the NFCG interventions, the BDI scores for the participants who received NFCG intervention, showed a considerable reduction in their levels of depressive symptoms, whilst the CG members had an increase. CBT combined with social skills and problem-solving has been shown to have improved the cognitive insight and functioning of Schizophrenic patients. ${ }^{22}$ Activity therapies are also techniques which use manual, recreational and creative art therapies to increase the patient's personal experiences, and the social responses. Psychiatric nurses at all levels may lead such groups or use the standard operating principles to reach beyond verbal communication with patients. ${ }^{22}$

Training nurses in CBT has also been found to be cost effective. Teaching CBT to home-visiting nurses was shown to improve clinical-practice and reduce urgent consultations with patients. 5 Individual and group-cognitive-behavioural therapies for moderately or severely depressed adults (BDI 2 14) were compared with each other in effectiveness, and both are superior, to providing no treatment at all. Individual cognitive therapy is equal to or better than tricyclic antidepressant drugs given at recommended therapeutic dosages, for depressed patients with a mean BDI of $30 .{ }^{23}$ In another study, group CBT, group counseling, and individual counseling interventions effectively reduced depression and anxiety in women with postnatal depression compared to routine care at 12 weeks; CBT was assessed as effective as counseling. However, individual counseling was more effective than group counselling. ${ }^{24}$ A study conducted among college women to test the effectiveness of cognitive behavioural therapy group intervention over a 6 week period, found that, compared to those in the control group, depressive symptoms and negative thinking had decreased and self-esteem had increased. ${ }^{25}$ A community-based intervention conducted among low-income-single-mothers using cognitive behavioral interventions prior to the onset of depression in at-risk individuals, showed that negative thinking was reduced and thought stopping and affirmations were useful. ${ }^{26}$

Nurses are in key positions to provide strategies to assist patients in safely integrating treatments, using mental health care plans. Nurses should use their communication skills when talking to patients and to particularly talk to them with their families in order to bring about a more satisfactory treatment plan that is mutually acceptable and mindful of patient's values and preferences. All health professionals should be knowledgeable regarding the spectrum and type of health-care required and requested by the patient or the family. ${ }^{2}$

\section{Study Limitations}

The sample size was small, and therefore the results may be limited in application. There was only one race group and one gender used as participants. The presence of children was not anticipated and consequently their attendance was not adequately planned for, however, they arrived at the sessions with their mothers and grandmothers. The use of antidepressants and the 'usual' or 'standard treatment' was not analysed and described fully, and this warrants further in-depth investigation and discussion.

\section{Conclusion}

The NFCG intervention as an adjunct to antidepressant medication (standard treatment), was effective in reducing the levels of depression. The women learnt to problemsolve, to support each other, build a positive bond with each other, develop new coping skills, and relieve themselves of some of the guilt, loss and grief that they had experienced. Given the challenges facing the health system, particularly in relation to shortages of specialists as well as the prevalence of depression, it is strongly advised that greater attention to such interventions be considered by psychiatric nurses. It is suggested that a replication of this study be done, using a multi-cultural-sample, more than one gender and a larger sample size to generalize the outcome of this study to other groups and settings.

\section{References}

1. Uys LR, Middleton L. Mental health nursing - a South African perspective. 5th edition. Lansdowne, Cape Town: Juta \& Co Ltd, 2010.

2. Hsu MC, Moyle W, Creedy D, Venturato L, Ouyang WC, Tsay SL. Use of antidepressants and complementary and alternative 
medicine among outpatients with depression in Taiwan. Arch Psychiatr Nurs 2009; 23:75-85.

3. World Health Organisation. The World Health Report. Mental Health: New Understanding, New Hope. Burden of Mental and behavioural Disorders. Geneva, 2001.

4. Tsai WP, Lin LY, Chang WL, Chang HC, Chou MC. The effects of the suicide awareness program in enhancing community volunteers' awareness of suicide warning sign.s. Arch Psychiatr Nursing 2010; 24:63-68.

5. Myhr G, Payne K. Cost-effectiveness of cognitive-behavioural therapy for mental disorders: Implications for Public Health Care Funding Policy in Canada. Can J Psychiatry 2006; 51:662-70.

6. Kuyken W, Dalgleish T, Holden ER. Advances in cognitivebehavioural therapy for unipolar depression. Can J Psychiatry 2007; 52:5-13.

7. Revicki DA, Siddique J, Frank L, Chung JY, Green BL, Prasad M, Miranda J. Cost-effectiveness of evidence-based-pharmacology or cognitive-behavior-therapy compared with community referral for major depression in predominantly low-income minority women. Arch Gen Psychiatry 2005; 62:868-875.

8. Murphy SA, Clark-Johnson L. Methodological issues associated with intervention research. Arch Psychiatr Nurs 2006; 20(6): 278-281.

9. Wheeler K. Psychotherapy for Advanced Practice Psychiatric Nurses. 3rd edition.St. Louis, Missouri: Mosby-Elsevier, 2008.

10. Moultrie A, Kleintjies S. Women's Mental Health in South Africa. South African Health Review: Durban, South Africa: Health System Trust 2006; 21: 347-366.

11. Zuma SM. Are we really transforming? Nursing Update 2003; 45.

12. Buys R, Muller $M$. The experiences and perceptions of nursing service managers regarding transformation of health services in selected provincial academic health complexes. Curationis 2000; 23: 50-56.

13. Luty J, O'Gara C. Validation of the 13-Item Beck Depression Inventory in alcohol-dependent people. Int J Psychiatry Clin Pract 2006; 10: 45-51.

14. Lawson B. Personal communication with chief professional nurse.
Regional Community Psychiatric Services Office, Department of Health, Durban, 16 March 2004.

15. Baumann SE. Primary health care psychiatry: A practical guide for Southern Africa. Kenwyn: Juta \& Co Ltd, 2007.

16. Domar AD, Clapp D, Kessel B, Orav J. The impact of group psychological interventions on distress in infertile women. Health Psychol 2000; 19: 568-575.

17. American Psychiatric Association. DSM IV-Diagnostic and Statistical Manual of Mental Disorders. 4th edition. Washington DC: American Psychiatric Association, 2000

18. McDowell I, Newell C. Measuring health-guide to rating scales and questionnaires. 2nd edition. New York: Oxford University Press, 1996.

19. Kavan MG, Pace TM, Ponterotto JG, Barone EJ. Screening for depression: use of patient questionnaires. Am Fam Physician 1990; 41:897-904

20. Gordon VC (unpublished). Facilitator's Manual and Women's Workbook. Minnesota: University of Minneapolis, 1988.

21. Gordon VC. Insight: A cognitive enhancement program for Women Workbook, 2002. Available at: http://www.insightforwomen.com/wresearch-ops.htm (Accessed 25/5/2011)

22. Kneisl CR, Trigoboff E. Contemporary Psychiatric-Mental Health Nursing. 2nded. New Jersey, USA: Pearson Prentice Hall, 2009.

23. Hodgkinson B, Evans D, O'Donell A, Walsh K. Comparing the effectiveness of individual therapy and group therapy in the treatment of depression: systematic review. Adelaide: Joanna Briggs Institute for Evidence Based Nursing and Midwifery; 1999.

24. Milgrom J, Negri LM, Gemmill AW. A randomized controlled trial of psychological interventions for postnatal depression. Br J Clin Psychol 2005; 44:529-542.

25. Peden AR, Hall LA, Rayens MK, Beebe LL. Reducing negative thinking and depressive symptoms. Journal of Nursing Scholarship 2000; 32(2):145-158.

26. Peden AR, Rayens, MK, Hall LA. A community based depression pre intervention with low income single mothers. Journal of American Psychiatric Nurses Association 2005; 11 (1):18-25

\section{INSTRUCTIONS FOR AUTHORS African Journal of Psychiatry (AJP)}

\section{WWw.ajop.co.za}

All manuscripts and correspondence should be emailed to:

Professor CP Szabo,

email: christopher.szabo@wits.ac.za 